C-A/AP/\#155 June 2004

\title{
Beam-Beam Studies For The Electron-Ion Collider ERHIC
}

\author{
C. Montag
}

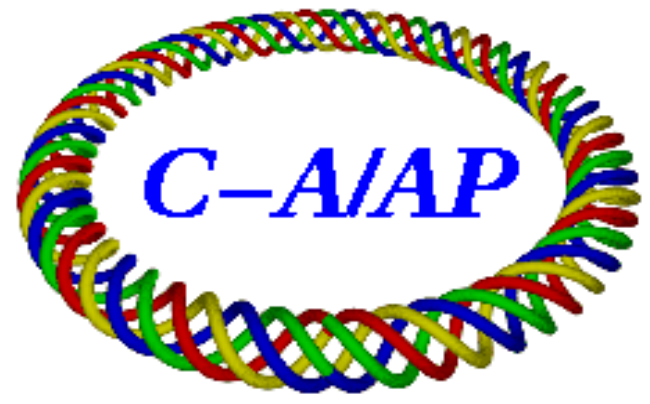

Collider-Accelerator Department Brookhaven National Laboratory Upton, NY 11973 


\title{
BEAM-BEAM STUDIES FOR THE ELECTRON-ION COLLIDER ERHIC *
}

\author{
C. Montag, Brookhaven National Laboratory, Upton, NY 11973, USA
}

\begin{abstract}
The electron-ion collider eRHIC [1] currently under study at BNL consists of a $10 \mathrm{GeV}$ electron storage ring to be added to the existing RHIC complex to study collisions of polarized electrons and relativistic heavy ions or polarized protons. To achieve high luminosities in the range of several $10^{32} \mathrm{~cm}^{-2} \mathrm{sec}^{-1}$, beam-beam tuneshift parameters of up to 0.08 are required for the electron beam. Simulation studies are performed to study the feasibility of these high tuneshift parameters. Recent results of these studies are presented.
\end{abstract}

\section{INTRODUCTION}

To study electron-ion collisions, the possibility of adding an electron ring to the existing RHIC facility is currently under study, with the electron ring circumference being $1 / 3$ of the RHIC circumference. Some basic machine parameters of this facility are listed in Table 1.

Beam-beam tuneshift parameters of up to 0.08 are required for the electron beam to achieve a luminosity of several $10^{32} \mathrm{~cm}^{-2} \mathrm{sec}^{-1}$. These high tuneshift parameters require careful simulation studies to ensure the feasibility of attaining the projected luminosity. Additionally, the unequal circumferences of the two rings lead to additional resonances [2] that have to be avoided when choosing the working point of the machine.

\section{UNEQUAL CIRCUMFERENCES}

Colliding bunched beams stored in machines of different circumference results in additional resonances that need to be avoided to ensure stable beam operation. While the resonance condition is

$$
Q_{1}-\frac{C_{1}}{C_{2}} Q_{2}=n, \quad n \text { integer },
$$

with $Q_{1}$ and $Q_{2}$ being the fractional tunes of the two machines and $C_{1}$ and $C_{2}$ denoting their respective circumferences, the actual width of these resonances is most easily studied by simulations. In the case of eRHIC, $C_{1} / C_{2}=3$, so each electron bunch collides with three different hadron bunches.

The simulation is performed by describing both beams as consisisting of rigid, gaussian bunches - one electron bunch and three hadron bunches. The beam-beam interaction is modelled as a mutual weak-strong kick, while the actual

\footnotetext{
${ }^{*}$ Work performed under the auspices of the U.S. Department of Energy
}

\begin{tabular}{lr}
\hline \hline electrons: & \\
ring circumference [m] & 1278 \\
number of bunches & 120 \\
geometric emittance hor./vert. [nm] & $53 / 9.5$ \\
$\beta$ functions hor./vert. [m] & $0.19 / 0.27$ \\
particles/bunch & $1.0 \cdot 10^{11}$ \\
beam-beam tune shift hor./vert. & $0.027 / 0.08$ \\
damping times hor./vert./long. [turns] & $1740 / 1740 / 870$ \\
\hline hadrons: & 3834 \\
ring circumference [m] & 360 \\
number of bun ches & $9.5 / 9.5$ \\
geometric emittance hor./vert. [nm] & $1.08 / 0.27$ \\
$\beta$ functions hor./vert. [m] & $1.0 \cdot 10^{11}(\mathrm{p})$, \\
particles/bunch & $1.0 \cdot 10^{9}(\mathrm{Au})$ \\
& $0.007 / 0.0035$ \\
\hline beam-beam tune shift hor./vert. & $100 / 50$ \\
\hline beam spot size hor.vert. $[\mu \mathrm{m}]$ & $4.4 \cdot 10^{32}$ \\
\hline luminosity [cm ${ }^{-2}$ sec $^{-1}$ ] & \\
\hline
\end{tabular}

Table 1: Interaction region parameters of the electron-ion collider eRHIC.

accelerator is described by a linear one-turn matrix. To seed the possible resonance, the electron bunch is started with an offset of $1 \mu \mathrm{m}$ at the interaction point (IP), which is small compared to the rms beam size of $\sigma_{x}=100 \mu \mathrm{m}$ horizontally and $\sigma_{y}=50 \mu \mathrm{m}$ vertically. Bunches are tracked for $3 \cdot 10^{4}$ electron beam turns, which corresponds to $10^{4}$ hadron beam turns in RHIC. Stability is defined as no increase of the electron beam amplitude during tracking.

As Figure 1 indicates, the additonal resonances reduce the stable tune space in one plane by some 30 percent. Additionally, electron tunes above .35 are no longer stable for a beam-beam tuneshift parameter of $\xi=0.08$, as it is required in the vertical plane.

Taking into account both planes and fixing the ion beam tunes to $Q_{p, x}=.21, Q_{p, y}=.23$, a sufficiently large region of the electron beam tune space $.06<Q_{e, x}<.34$, $.05<Q_{e, y}<.28$ leads to a stable situation, as shown in Figure 2.

\section{DYNAMIC FOCUSING}

With a beam-beam tuneshift parameter as high as $\xi=0.08$, the beam-beam interaction has a significant effect on the entire dynamics and cannot be treated as a small perturbation. For instance, the resulting small amplitude 


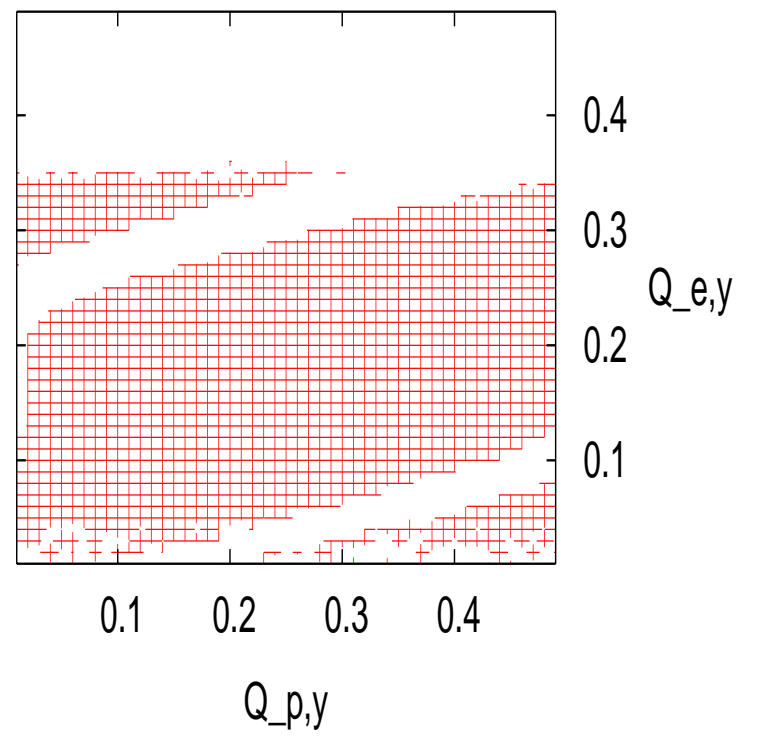

Figure 1: Tune diagram $Q_{e, y}$ vs. $Q_{p, y}$ in the vertical plane, for a beam-beam parameter of $\xi=0.08$. The checkered areas indicate stable tune regions.

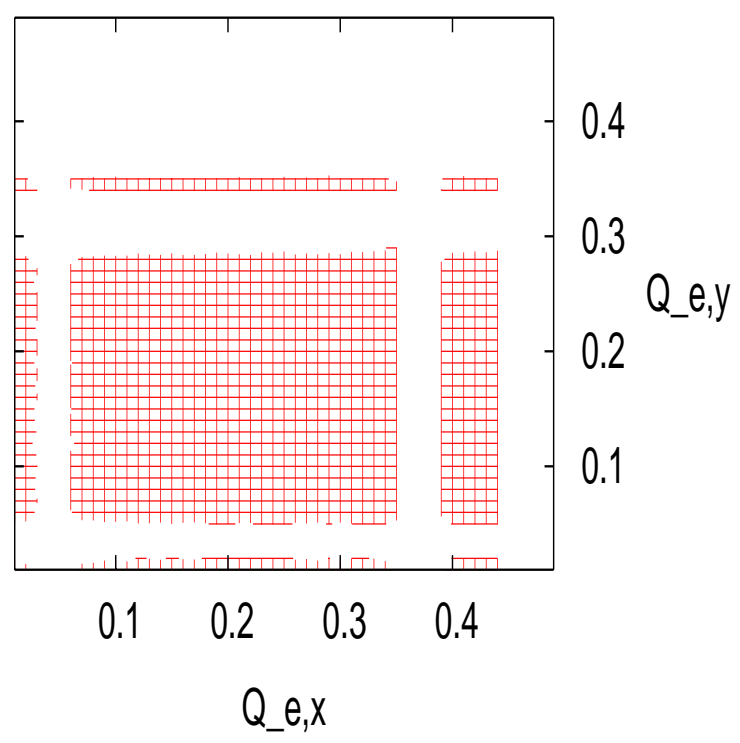

Figure 2: Electron beam tune diagram, $Q_{e, y}$ vs. $Q_{e, x}$, for a hadron working point of $Q_{p, x}=.21, Q_{p, y}=.23$. The checkered areas indicate stable tune regions.

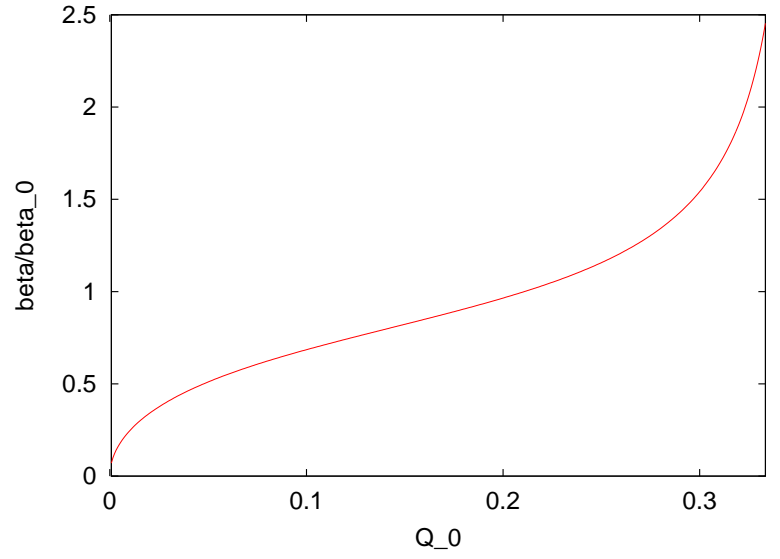

Figure 3: Dynamic $\beta / \beta_{0}$ vs. base tune $Q_{0}$ for a beam-beam parameter $\xi=0.08$, calculated according to Equation (3).

tune $Q$ is no longer simply the sum of the machine base tune $Q_{0}$ and the beam-beam tuneshift $\xi$, but is expressed as

$$
\cos (2 \pi Q)=\cos \left(2 \pi Q_{0}\right)-2 \pi \xi \sin \left(2 \pi Q_{0}\right) .
$$

Likewise, the $\beta$-function at the interaction point gets significantly altered due to the strong beam-beam lens at the IP, resulting in a tune-dependent $\beta$-function,

$$
\beta=\frac{\beta_{0}}{\sqrt{1+4 \pi \xi \cot \left(2 \pi Q_{0}\right)-4 \pi^{2} \xi^{2}}} .
$$

As Figure 3 shows, $\mathrm{x}$ the resulting $\beta$-function at the IP is significantly reduced for tunes just above the integer, which provides additional focusing. Therefore, these tunes are generally preferred.

The presence of this strong beam-beam lens modifies the entire machine optics and therefore the equilibrium beam emittance, which depends on the "curly $\mathrm{H}$ " function

$\mathcal{H}(s)=\beta(s) D^{\prime 2}(s)+2 \alpha(s) D(s) D^{\prime}(s)+\gamma(s) D^{2}(s)$,

where $\alpha(s), \beta(s)$, and $\gamma(s)$ are the Twiss parameters at location $s$, while $D(s)$ is the dispersion in the same location, and $D^{\prime}(s)=\mathrm{d} D(s) / \mathrm{d} s$. The resulting dynamic emittance $\epsilon$ can be approximated as [3]

$$
\epsilon \approx \frac{1+2 \pi \xi \cot \left(2 \pi Q_{0}\right)}{\sqrt{1+4 \pi \xi \cot \left(2 \pi Q_{0}\right)-4 \pi^{2} \xi^{2}}} \epsilon_{0},
$$

where $\epsilon_{0}$ refers to the equilibrium emittance of the unperturbed lattice, but in fact depends rather strongly on the actual machine lattice [4]. For the simulation, the resulting equilibrium emittance is therefore calculated according to the respective actual dynamic machine lattice for each working point.

\section{SIMULATION RESULTS}

The nonlinear eRHIC electron ring lattice [1] is optimized for a working point of $Q_{x}=.10, Q_{y}=.14$ and 

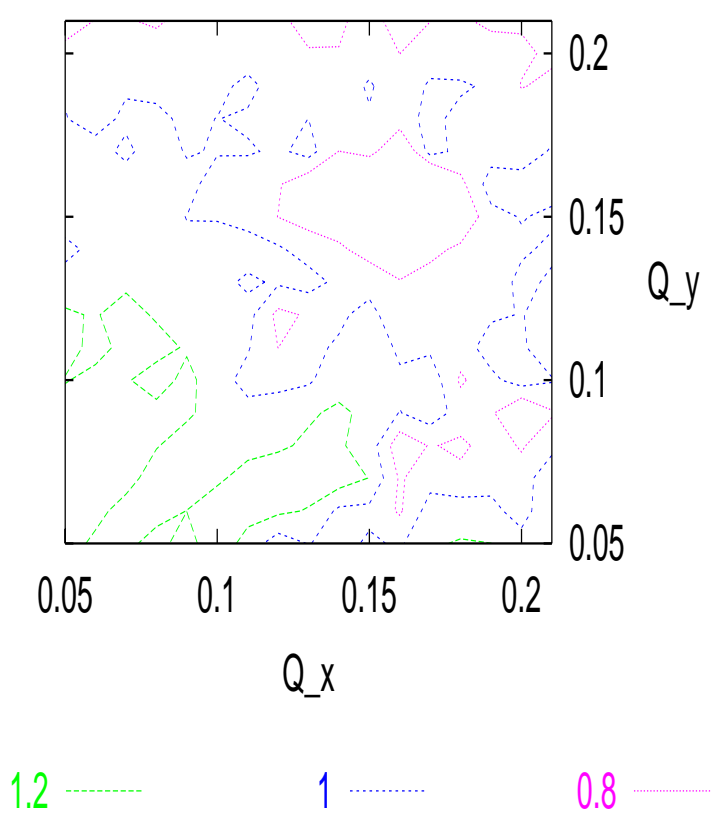

Figure 4: Contour plot of the resulting normalized luminosity $\mathcal{L} / \mathcal{L}_{0}$ vs. horizontal and vertical electron beam tune. $\mathcal{L}_{0}$ denotes the geometric design luminosity according to Table 1 .

zero chromaticity in both planes. Tracking studies were performed to find the best working point for this machine. Tunes are scanned in the range between the integer and the quarter resonance by adjusting the main quadrupoles accordingly, and for each working point the chromaticities are readjusted to zero.

The beam is represented by 100 macroparticles with zero momentum deviation. These particles are tracked for ten radiation damping times, including quantum excitation and radiation damping. The horizontal equilibrium emittance is adjusted according to the radiation integrals that correspond to each individual working point, while the vertical equilibrium emittance is assumed to be unaffected by the machine tune. The resulting equilibrium luminosity is calculated according to the obtained rms beam sizes $\sigma_{x}$ and $\sigma_{y}$ after tracking for ten damping times. Figure 4 shows a contour plot of the resulting luminosity in units of the nominal, geometric luminosity, as a function of the working point $\left(Q_{x}, Q_{y}\right)$.

While the luminosity generally increases with lower tunes, the coupling resonance is clearly visible in this plot, as is the 6 th order resonance in both planes. However, to ensure proper matching of beam sizes of the hadron and electron beam at the IP, both planes have to be checked separately. Figures 5 and 6 depict the resulting rms beam sizes in the two planes as a function of the working point. While in the horizontal plane a significant beam size increase occurs mostly along the 6 th order resonance line, the situation in the vertical plane is more complex due to the larger beam-
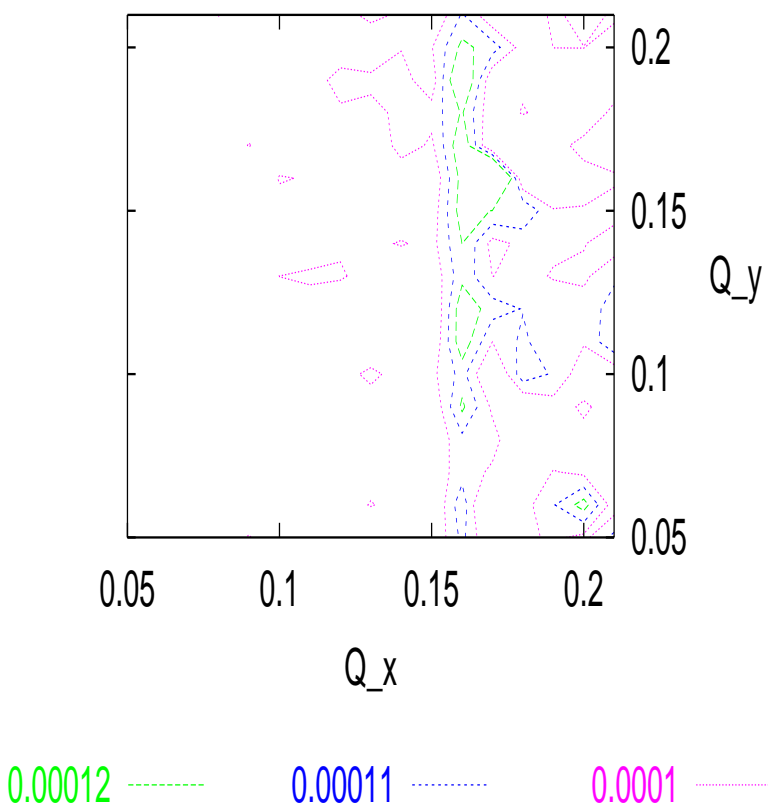

Figure 5: Contour plot of the horizontal rms electron beam size $\sigma_{x}$ (in meters) vs. electron beam working point $\left(Q_{x}, Q_{y}\right)$.

beam parameter.

\section{CONCLUSION}

Simulation studies show that even with beam-beam tuneshift parameters of up to $\xi=0.08$, sufficiently large areas in the working diagram can be found that support the projected luminosity performance of eRHIC. These simulations also indicate that a luminosity significantly higher than the geometrical one could be achieved by moving the electron beam tunes very close to the integer, thus taking advantage of dynamic focusing effects. However, this results in a beam size mismatch of the two beams, which may cause emittance deterioration of the ion beam.

According to simulations, unequal circumferences of the two rings are not much of a concern in terms of barycenter motion of the two beams, as long as certain additional resonances are avoided. The remaining stable tune space is sufficiently large to ensure stable operation of the electronion collider eRHIC. The location of the stable area within the tune diagram depends on the working point chosen for the ion ring. For the present RHIC working point, stable electron tunes are consistent with those found necessary to achieve design luminosity.

\section{REFERENCES}

[1] eRHIC Zeroth-Order Design Report, BNL note C-A/AP/142

[2] K. Hirata and E. Keil, "Barycenter motion of beams due to beam-beam interaction in asymmetric colliders", Nucl. Instr. and Meth. A 292 (1990) 156-168 

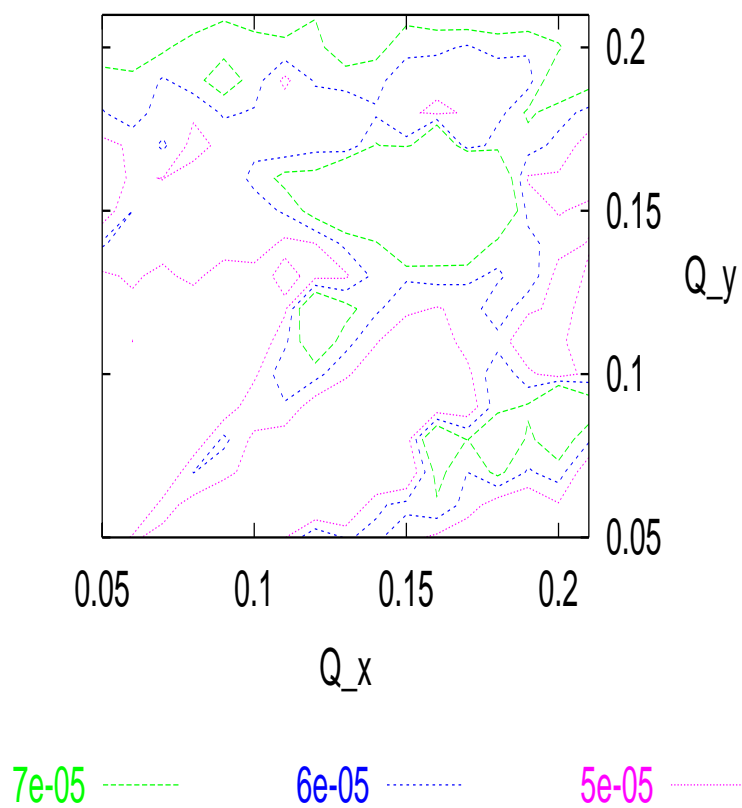

Figure 6: Contour plot of the vertical rms electron beam size $\sigma_{y}$ (in meters) vs. electron beam working point $\left(Q_{x}, Q_{y}\right)$.

[3] K. Hirata, "Beam-beam effects", in: A. W. Chao and M. Tigner (eds.), Handbook of Accelerator Physics and Engineering, World Scientific, Singapore 1999

[4] A. V. Otboyev and E. A. Perevendentsev, "Self-consistent $\beta$ functions and emittances of round colliding beams", Phys.

Rev. ST Accel. Beams 2, 104401 (1999) 